\title{
Uma abordagem hermenêutica da relação saúde-doença
}

\author{
An hermeneutic approach \\ to health-disease relationship
}

Andrea Caprara 1

1 Departamento de Saúde Pública, Centro de Ciências da Saúde, Universidade Estadual do Ceará. Av. Paranjana 1700 , Fortaleza, CE 60740-000, Brasil. a.caprara@flashnet.it

\begin{abstract}
This work aims to contribute to the current discussion on the relationship between health and disease, taking a hermeneutic perspective on the issue. The first section, referring to the discussion among the hermeneutic, phenomenological, and existential lines of philosophy, analyzes the work of two philosophers, Kierkegaard and Heidegger, who profoundly influenced contemporary hermeneutics. The article discusses the concept of anguish, which for Kierkegaard (contrary to the biomedical approach) is a constitutive component of human beings: for Kierkegaard, as subsequently for Heidegger, anxiety is not a pathological symptom but a state that allows for privileged access to self-knowledge. In the second section, we approach how hermeneutics, and especially the work of Gadamer, developed the concepts of health, illness, and suffering; we also analyze how in recent years this perspective has influenced the social sciences (and particularly medical anthropology) in their approach to health problems. The third and final section discusses the implications of hermeneutics for clinical training and practice, demonstrating the applicability of Heidegger's and Gadamer's thinking for work by physicians and nurses.
\end{abstract}

Key words Medical Philosophy; Medical Antropology; Health-Disease Process

Resumo Este trabalho pretende contribuir para a discussão presente sobre a relação entre a saúde e a doença, abordando a problemática por meio da perspectiva hermenêutica. Na primeira parte, retomando a discussão que foi se desenvolvendo dentro das linhas filosóficas hermenêutica, fenomenológica e existencial, será analisada a obra de dois filósofos, Kierkegaard e Heidegger, que influenciaram profundamente a hermenêutica contemporânea. Será apresentado o conceito de angústia que, ao contrário da abordagem biomédica, para Kierkegaard é um componente constitutivo dos seres humanos: para este autor, assim como sucessivamente para Heidegger, a ansiedade não é um sintoma patológico mas um estado que permite um acesso privilegiado de autoconhecimento. Na segunda parte, tentaremos abordar como, na hermenêutica, e principalmente na obra de Gadamer, foram sendo desenvolvidos os conceitos de saúde, doença, sofrimento; analisaremos também como esta perspectiva influenciou, nos últimos anos, as ciências sociais que abordam os problemas de saúde, em particular a antropologia médica. Na terceira e última parte, apresentaremos as implicações da hermenêutica na formação e prática clínica, mostrando a aplicabilidade do pensamento de Heidegger e Gadamer no trabalho de médicos e enfermeiros.

Palavras-chave Filosofia Médica; Antropologia Médica; Processo Saúde-Doença 


\section{Introdução}

Em muitos países ocidentais e da América Latina, apesar de um processo de crescimento econômico, estamos assistindo a situações em que as desigualdades sociais e em saúde persistem, até aumentam, mostrando uma real incapacidade da sociedade ocidental em eliminar a pobreza. A crise do modelo econômico ocidental foi, nos anos 70 e 80 , objeto de análise de diferentes estudos: para Ricoeur (1994), por exemplo, a crise é uma condição permanente e estrutural de nossa sociedade que se reflete nos estudos humanísticos, na filosofia, na história, nas ciências sociais, na antropologia.

Na saúde pública, desde a metade do século XX, diferentes autores como McKeown (1979), demostraram que, se a pobreza não é causa direta da doença é o principal determinante. Esta abordagem que mostra que as condições de saúde estão ligadas a fatores macroeconômicos e sociais não é idéia do século XX. Rudolph Virchow, na metade do século XIX, afirmava a necessidade de lutar contra as desigualdades sociais, origem principal das doenças, assim como Villermé na França demonstrava em 18201830 as diferenças das taxas de mortalidade entre as classes ricas e pobres (Virchow, 1985).

A influência dos fatores macrossociais e do contexto político-econômico sobre as condições de saúde da população e da própria prática médica, foi objeto nos anos 60-70, das abordagens marxistas da medicina social (Navarro, 1983). Nos mesmos anos e com uma perspectiva similar desenvolveram-se a epidemiologia crítica, chamada também epidemiologia social (Breilh \& Granda, 1989), os estudos sobre o impacto da estrutura de classe na organização dos serviços de saúde (Laurell, 1989), a antropologia médica crítica (Frankenberg, 1988). Essas perspectivas críticas da ideologia médica, tiveram a influência dos pensadores marxistas, assim como de autores como Foucault (1977), e da própria Escola de Frankfurt (Benjamin et al., 1983), colocando uma atenção particular a conceitos relativos ao poder, à hegemonia, à resistência, à prática da medicina moderna e ao desenvolvimento de tecnologias.

Sem negar a importância desta abordagem, a saúde nas ciências sociais e na antropologia em particular, não é vista somente como conseqüência de fatores sociais e econômicos. Uma tradição importante interpreta a doença como um produto culturalmente determinado. Nesta perspectiva, a cultura, entendida como conjunto de idéias, conceitos regras, comportamentos compartilhados em um determinado grupo cultural, organiza a experiência da doença e do comportamento de maneira diversa nas diferentes sociedades (Bibeau, 1981; Good, 1994; Kleinman, 1988). A cultura produz estruturas simbólicas, metáforas e outras figuras ligadas à doença. Assim como colocado por Birman (1991), em todas as sociedades o real é transformado em uma rede simbólica de significados, base fundamental de todo processo experiencial. Tal abordagem interpretativa procura entender o significado dos comportamentos, das ações dos indivíduos influenciados por autores pertencentes à tradição hermenêutica como Gadamer (1997) e Paul Ricoeur (1994). A experiência do indivíduo coloca-se no primeiro plano da produção dos discursos científicos, dentro de uma perspectiva que procura o significado das ações na relação entre interpretante e interpretado, tentando superar a distinção entre sujeito e objeto na pesquisa científica (Geanellos, 2000).

Este trabalho pretende contribuir para a discussão presente sobre a relação entre a saúde e a doença (Almeida Filho, 2001), abordando a problemática por meio da perspectiva hermenêutica. Com essa finalidade, propõe-se explorar o conceito de saúde segundo três sucessivos desdobramentos: (1) na primeira parte, retomando a discussão que foi se desenvolvendo dentro das linhas filosóficas hermenêutica, fenomenológica e existencial, será analisada a obra de dois filósofos, Kierkegaard e Heidegger, que influenciaram profundamente a hermenêutica contemporânea. No campo da saúde, a obra destes autores é importante pela diferente forma de abordar os problemas em comparação com a perspectiva biomédica. Por exemplo será apresentado o conceito de angústia que, ao contrário da abordagem biomédica, para Kierkegaard (1952, 1991), é um componente constitutivo dos seres humanos: para este autor, assim como sucessivamente para Heidegger (1976), a ansiedade não é um sintoma patológico mas um estado que permite um acesso privilegiado de autoconhecimento. $\mathrm{Na}$ perspectiva hermenêutica contemporânea, o estado de ansiedade revela a procura de um novo significado de vida; trata-se de um problema existencial, não somente de um problema biológico ou comportamental, e permite abordar a relação saúde-doença por meio de um novo olhar. Nesse sentido, acreditamos que o conceito de ansiedade, angst, merece hoje um renovado interesse, seja de um ponto de vista médico ou filosófico. Acaba-se colocando, na interseção entre saúde e doença, nos limites das definições, constituindo um espaço de reflexão filosófica com importantes implicações no campo da prática médica. (2) Na segunda 
parte, tentaremos abordar como, na hermenêutica, e principalmente na obra de Gadamer, foram sendo desenvolvidos os conceitos de saúde, doença, sofrimento; o trabalho de Gadamer, filósofo alemão, considerado hoje pai da hermenêutica contemporânea, foi influenciado por Kierkegaard e por Heidegger (Schleibel, 2000). A saúde, segundo Gadamer (1994), fica escondida por boa parte do tempo, revelandose através do bem-estar. Nesse estado, esquecemos de nós e somente nos momentos de cansaço, de esforço, de fadiga, lembramos de novo do nosso estado. Nesse sentido, para Gadamer (1994), a saúde coincide com o ser no mundo, com a satisfação de ter uma vida ativa. A perspectiva hermenêutica permite uma nova construção de modelos de saúde-doença, que recuperam a dimensão experiencial, assim como aquela psicossocial. (3) Na terceira e última parte, apresentaremos as implicações da teoria interpretativa na formação e prática clínica, mostrando a aplicabilidade do pensamento de Heidegger e Gadamer no trabalho de médicos e enfermeiros (Dreyfus \& Zimmerman, 1991; Svenaeus, 1999; Wiklund et al., 2002). A abordagem hermenêutica da medicina nos permite explorar a experiência humana da doença, entender como os pacientes lidam com suas experiências específicas frente aos médicos, em relação à saúde, à doença e ao sofrimento (Robertson-Malt, 1999). Os pacientes, tradicionais “objetos" da prática médica, são na verdade indivíduos que vivem, refletem e que, juntos com o médico, transformam o encontro clínico (Evans \& Sweeney, 1999). Nos últimos anos foi se desenvolvendo uma grande área de reflexão e pensamento denominada "humanidades médicas”, que pretende explorar como a experiência humana lida com outras experiências de pacientes, médicos, saúde, doença e sofrimento. Na formação em medicina, a abordagem das humanidades médicas prevê a incorporação de elementos das ciências humanas (filosofia, psicologia, antropologia, literatura) nos cursos de graduação e de especialização. Nesta concepção integrada das humanidades médicas, a medicina é entendida como forma de auto-exploração, reconhecendo que os componentes material e experiencial são fundidos entre eles (Evans \& Sweeney, 1999). As humanidades médicas pretendem não somente melhorar a relação médico-paciente, as capacidades comunicacionais dos médicos, mas também aprofundar a narrativa do paciente e procurar novas formas de promoção do bem-estar, reduzindo o impacto da doença e do sofrimento (More, 1976; Widdershoven, 1999). Enfim, temos de considerar a utilização da abordagem herme- nêutica na pesquisa qualitativa na área da saúde. Apesar da presença de numerosos métodos existentes, as premissas filosóficas comuns de Ricoeur e Gadamer constituem a base de diferentes abordagens na análise das narrativas (Geanellos, 2000; Robertson-Malt, 1999; Todres \&Wheeler, 2001).

\section{A terceira margem da saúde: a angústia em Kierkegaard e Heidegger}

O termo hermenêutica na filosofia grega expressa a arte de interpretar. Com o passar do tempo adquiriu um significado mais amplo, indicando, no âmbito filosófico, diversas formas de teoria da interpretação, entre as quais o existencialismo, a fenomenologia e a própria hermenêutica, que constituem diversas formas de expressão da filosofia continental. Os filósofos que pertencem a esta linha de pensamento se ocupam da existência humana, não do ponto de vista da observação, mas da reflexão filosófica. Nessa perspectiva, o homem é considerado não somente enquanto organismo biológico, mas algo mais, assim como a medicina é considerada algo mais do que a ciência natural.

Para esclarecer melhor a abordagem hermenêutica em relação à saúde e à doença, começaremos com um exemplo que se refere ao conceito de angústia (angst), tal como desenvolvido por Kierkegaard (1813-1855) e Heidegger (1889-1976). Para estes autores, a angústia deve ser considerada como elemento constitutivo dos seres humanos; em particular, para Kierkegaard (1952), a angústia constitui um estado fundamental da existência humana. Os seres humanos que não conhecem a melancolia têm um espírito que não conhece a metamorfose (Kierkegaard, 1952, 1972). Ao mesmo tempo, para Heidegger, a ansiedade não é um sintoma patológico, mas um estado que permite um acesso privilegiado de autoconhecimento (Heidegger, 1976).

Um caso clínico, citado por Wulff et al. (1995:152), ilustra com clareza este tema: "Uma mulher de 45 anos, aproximadamente, procura um especialista por problemas nervosos. Tratase de uma dona de casa, divorciada, que não consegue dar finalidade à sua própria existência. Os filhos são adultos, deixaram a casa, ela perdeu os amigos e não consegue sair de casa sozinha. Na maior parte do tempo encontra-se em um estado de ansiedade permanente. O psiquiatra escuta a história e em função de sua concepção de doença mental, caso ele siga a perspectiva biologista, poderá seguir uma terapia comportamental ou prescrever um ansiolí- 
tico". Para a perspectiva hermenêutica, ao contrário da abordagem biomédica, o estado de ansiedade da paciente revela a procura de um novo significado de vida; trata-se de um problema existencial, não somente de um problema biológico ou comportamental. Para os filósofos existencialistas, fenomenológicos e hermenêuticos o interesse se dirige aos problemas existenciais, interpretando as ações humanas. Colocaremos a questão principalmente em referência às angústias persistentes e que estimulam a procurar uma nova finalidade da existência.

As concepções de Kierkegaard sobre o homem são constitutivas para o desenvolvimento das idéias de filósofos existencialistas como Heidegger e Jean Paul Sartre. Heidegger (1977), afirma na obra Sein und Zeit, que Kierkegaard foi muito importante no desenvolvimento do conceito de angústia, angst Para Kierkegaard (1991), a angústia é um componente constitutivo dos seres humanos; quem não conhece a angústia não é um ser humano. Como afirma o mesmo Heidegger (1976), Kierkegaard aborda o conceito de uma perspectiva cristã enquanto que Heidegger faz isso desde uma perspectiva secular (Dreyfus, 1991). Para Heidegger a angústia serve como um transtorno que revela a natureza do Dasein, que poderíamos traduzir como: "existência humana cotidiana", de ser no mundo (Dreyfus, 1991).

Somente por meio de uma processo de reflexão filosófica é possível, para os filósofos da tradição hermenêutica, aprofundar nossos conhecimentos sobre as características constitutivas dos seres humanos. Também os estudos empíricos sobre os seres humanos permitem descrever o funcionamento e as ações humanas, mas estes dados têm de ser interpretados sucessivamente por meio da abordagem hermenêutica. No livro A Doença Mortal, Kierkegaard (1952) enfatiza como os seres humanos se relacionam com si mesmos e se percebem como seres que atuam livremente. Nesse sentido, os seres humanos podem ser considerados como uma conjunção entre dimensão biológica e psicossocial, em um processo de autoreflexão, de liberdade. Por isso, para Kierkegaard a angústia é a realidade da liberdade (Dreyfus, 1991). Nessa concepção, a angústia humana constitui um estado fundamental vinculado estritamente à capacidade de autoreflexão, de atuar livremente, identificando as possibilidades da liberdade (Dreyfus, 1991). Esta dimensão deve ser separada da esfera mental em que se manifestam os sentimentos como o medo, que são reações comuns e presentes também nos seres animais.
Heidegger (1976), em Ser e Tempo, aborda o problema da angústia, concordando com Kierkegaard de que se trata de um estado fundamental, mas afirma também que está vinculada à nossa compreensão e interpretação do universo. Para Heidegger a compreensão não é um fenômeno psicológico, mas um estado constitutivo dos seres humanos como a angústia.

O homem, para Heidegger, continuamente procura compreender/interpretar o significado de mundo. Quem não se coloca no mundo como ser interpretante perde a própria subjetividade (Wulff et al., 1995). A angústia nos indica que os significados do mundo mudaram e que os contatos com os outros seres humanos perderam seu próprio significado (Wulff et al., 1995). É neste sentido que para Heidegger a angústia permite o acesso à autoreflexão. Para a perspectiva hermenêutica, as pessoas que vivem um estado de ansiedade não somente têm de ser diagnosticadas de um ponto de vista clínico, mas é necessário que comecem um processo de reflexão sobre sua própria existência. Essa pessoa é livre para escolher, mesmo se decide não enfrentar esses problemas e utilizar somente ansiolíticos, mas nesse caso dificilmente poderá dar uma solução a seus problemas existenciais. Uma abordagem biomédica permite controlar a depressão, ou a ansiedade, ou a angústia por meio da utilização de psicofármacos eliminando ou reduzindo a sintomatologia, mas a perspectiva hermenêutica coloca-se de forma crítica frente a este modo de abordar os problemas. A utilização dos psicofármacos é aceita somente naquelas formas, tais como as psicoses maníaco-depressivas ou a esquizofrenia, em que a utilização de medicamentos é indiscutivelmente necessária.

Para os empiristas as conclusões da abordagem hermenêutica não podem ser demonstradas cientificamente, e não sendo consideradas nem verdadeiras nem falsas, são simplesmente sem sentido. Por outro lado, os hermeneutas afirmam que os empiristas colocam mal a questão porque também os resultados científicos têm de ser interpretados e que a maneira com a qual se aborda um problema tem uma influência sobre o método que será escolhido. É impossível utilizar sempre o mesmo método científico em diferentes áreas. Segundo Wulff et al. (1995:162-163): "a análise hermenêutica é um processo inicial indispensável porque não tem sentido um estudo empírico das propriedades de uma pessoa sem se ter analisado o conceito de pessoa. Por esta razão, a ciência natural é subordinada à reflexão hermenêutica". 


\section{Os conceitos de saúde e doença na obra de Gadamer e suas influências nas ciências sociais em saúde}

Estudos recentes mostram que não existe consenso entre os diferentes campos de saber sobre o conceito de saúde, doença, "normal” e patológico (Almeida Filho, 2001; Czeresnia, 1999). A incapacidade da epidemiologia, por exemplo, de tratar a categoria saúde, seria, segundo Almeida Filho (2001), indicador desta crise. Uma reflexão teórica importante sobre os conceitos de saúde e doença, e que gostaríamos de resgatar neste trabalho, tem sido desenvolvida dentro das linhas filosóficas da hermenêutica, do existencialismo e da fenomenologia. Em particular, Gadamer (1994), na coletânea de ensaios Uber die Verborgenheit der Gesundheit (na tradução em italiano: Dove si Nasconde la Salute), indica que, enquanto a doença chama nossa atenção pela sua presença, a saúde não desperta nosso interesse, ficando escondida.

Nessa situação, para o grande pensador alemão, a ciência médica teria de ser reposta como ciência da doença, porque é o estado de doença que, aparecendo, produz um sentimento de perigo e estimula uma resposta terapêutica. A prática médica, nesse sentido, tentando modificar o percurso da natureza, dominando suas manifestações patológicas, ao mesmo tempo deixa de lado um interesse sobre a concepção de saúde e, em parte, a prevenção das doenças (Gadamer, 1994). A medicina tentou estabelecer normas, valores que teriam de ser utilizados de forma universal, parâmetros que constituem uma convenção por meio da qual procura-se aproximar a realidade.

A medicina ocidental é considerada como área pertencente às ciências naturais e, nesse sentido, os seres humanos são analisados de um ponto de vista biológico; para a perspectiva hermenêutica (arte da interpretação), ao contrário, os indivíduos, além de seres biológicos, têm de ser considerados como sujeitos que refletem e vivenciam uma experiência subjetiva da doença. Para abordar esta questão, Gadamer (1994) coloca o problema de como um indivíduo tem de se orientar na vida em relação à saúde e à doença. Este autor coloca a diferença entre ciência médica e arte da cura, que corresponde à relação entre conhecimento e sua aplicabilidade. Por exemplo, para cada caso de patologia, como a esclerose múltipla, existem conhecimentos científicos baseados na evidência que sugerem esquemas de tratamento e de conduta; mas cada paciente é único e o médico tem de levar em conta isso, porque cada paciente vive a doença de forma diferente. Nesse sen- tido, a medicina baseada em evidências não é suficiente. Para Gadamer (1994), a diferença entre conhecimentos gerais e sua aplicabilidade é objeto de discussão e de estudo na hermenêutica. Os conhecimentos são adquiridos por meio do estudo, enquanto que sua aplicabilidade pode ser adquirida somente por intermédio de um longo processo experiencial.

Na concepção gadameriana, a saúde tem de ser entendida como equilíbrio; esta visão se aproxima da saúde entendida pelos utilizadores das diferentes formas de medicinas alternativas, medicinas das quais Gadamer mesmo é grande utilizador e seguidor. Ele valoriza conceitos típicos dessas formas de medicina como equilíbrio, harmonia, diálogo, globalidade. Ter cuidado com a própria saúde significa evitar os excessos, prevenindo o uso de medicamentos ou de exames de laboratório desnecessários. Para Gadamer, a saúde constitui o ritmo da vida, o processo pelo qual se produz o equilíbrio da respiração, do sono, do estar acordado etc. Nessa visão, Gadamer afirma que nós somos natureza e a natureza que está em nós ajuda a conservar esse equilíbrio. A saúde para Gadamer não pode ser mensurada porque está ligada ao estado de ser de cada indivíduo; é por isso que tem sentido perguntar às pessoas se elas se sentem doentes (Gadamer, 1994).

Procurando estabelecer uma comunicação entre medicina e filosofia ocidental, Gadamer (1994) afirma que a arte da cura tem aspectos que vão além da dimensão biológica, característica da abordagem médica, aspectos que não podem ser reduzidos à posição da ciência médica. Nessa abordagem ele não critica a medicina científica, mas tenta uma aprofundada análise dos conceitos chave que fundamentam o discurso médico: doença, cura, morte, relação médico-paciente. Ele propõe uma nova medicina "humanista" que utiliza os instrumentos técnicos e diagnósticos mas que ao mesmo tempo analisa o ser humano na sua totalidade, o seu ser no mundo.

Estas idéias influenciaram a dimensão socioantropológica, principalmente em autores como Clifford Geertz (1983), Arthur Kleinman (1988) e Byron Good (1994), nos Estados Unidos; Gilles Bibeau (1992) e Ellen Corin (1992), no Canadá e diferentes autores da antropologia médica européia: lembramos por exemplo Angel Martínez-Hernáez (1998), na Espanha e Mariella Pandolfi, na Itália (1990). 
Teoria interpretativa e teoria crítica no campo da saúde: rumo para uma nova abordagem teórica?

Para os autores críticos, as culturas não são somente sistemas de significados, mas ideologias que mascaram iniqüidades políticas e econômicas. Para Mishler (1984), por exemplo, que aplica a Teoria da Ação Comunicativa de Habermas (Habermas, 1984), no estudo da relação médico-paciente, a abordagem médica fragmenta e dificulta a expressão da voz dos pacientes (Barry et al., 2001). As influências da Escola de Frankfurt e de Jurgen Habermas são evidentes e refletem discussões similares desenvolvidas no campo filosófico (Habermas, 1976). Habermas afirma que as descrições do processo hermenêutico de Gadamer não oferecem garantias suficientes e que há uma compreensão distorcida devido às pré-compreensões falsas. A sociedade está caracterizada por estruturas de poder, interesses que influenciam a compreensão de nós mesmos e dos outros. A consciência histórica não é suficiente para prevenir distorções ideológicas. A ideologia é vista como uma teoria a serviço de interesses de grupos particulares, que contribui na consolidação dos equilíbrios existentes. As ideologias fornecem, portanto, uma visão distorcida da realidade (Habermas, 1976).

Como afirma Corin (1995), poucos são os estudos no campo da saúde que hoje tentam combinar a perspectiva interpretativa, influenciada pela tradição hermenêutica, com a teoria crítica e, apesar das diferenças existentes, elas têm uma série de elementos em comum: ambas analisam a cultura como uma realidade "pervading”, uma dimensão dinâmica, que não pode ser expressada como simples variável a ser estudada com variáveis estatísticas. O segundo elemento em comum entre essas perspectivas é a abordagem transcultural; segundo Corin as perspectivas transculturais são as únicas que nos permitem descobrir a relatividade de nossos conceitos e teorias, e ajudam a "ampliar" nosso conceito de realidade (Corin, 1995).

Gilles Bibeau (1992), no seu ensaio Entre Sens et Sens Commun, nos convida a refletir, como ponto de partida, sobre os elementos metafóricos e empíricos do pensamento humano. Este autor enfatiza que é necessário articular o saber e o significado: "A procura do significado e a atribuição do sentido constituem o percurso fundamental que carateriza as culturas humanas, percurso que inscreve o sentido até o centro das classificações e do estabelecimento da ordem empírica do mundo operada pelo senso comum" (Bibeau, 1992:89).
Com a referência de três autores, Bourdieu (1980), Geertz (1983) e Tambiah (1985), que analisaram o papel da prática cotidiana nos processos de conhecimento, Bibeau (1992) enfatiza o papel chave que a experiência comum tem na construção dos saberes: o sentido comum apresenta um caráter prático e coincide largamente com o que poderíamos chamar de sabedoria popular. Para Geertz (1983), é a análise das ações cotidianas que vai permitir definir os códigos que estruturam o pensamento e conferem um significado ao mundo: para Geertz, as ações cotidianas formam aqui o que ele define como o senso comum que permite uma leitura direta da realidade.

Com base nessas considerações, Corin et al. (1993) desenvolveram um modelo semânticopragmático que objetiva conjugar as perspectivas hermenêutica e crítica (Bibeau, 1994). Esta abordagem tenta analisar a problemática por meio de três níveis de análise: (a) um estudo do saber, focalizando a atenção sobre os discursos, as narrativas dos membros da comunidade este primeiro nível procura identificar a semiologia, os signos, os sinais, os indicadores com base nos quais os diferentes aspectos de saúde e doença são percebidos na comunidade; (b) uma análise do sistema de interpretação; (c) um terceiro nível de análise, chamado sistema de ação, visa a entender as respostas, as reações para procurar respostas aos problemas. Mas para superar os perigos de uma análise do sujeito, da microrealidade, é necessário, segundo Bibeau (1994), considerar também os fatores macrossociais, situando-nos em uma dimensão do coletivo; a reconstrução de casos individuais terá de ser complementada por uma análise do espaço social, em uma análise da interação entre histórias e casos individuais e processos coletivos. Bibeau sugere nesse sentido uma abordagem em dois níveis: (a) uma primeira análise das "condições estruturantes" que se referem ao desenvolvimento econômico, político e social e das condições cotidianas de vida; (b) uma segunda que explora as experiências organizadoras coletivas, os elementos sociosimbólicos de um grupo, seu próprio sistema de valores. Este processo tem como objetivo identificar os fatores negativos que fragilizam um grupo, assim como os fatores protetores: precisa identificar as estratégias coletivas de proteção, paralelamente a uma análise dos fatores de risco e de fragilização.

Uma perspectiva teórica similar, que tenta combinar as duas perspectivas, foi desenvolvida por Good (1994), no seu livro Medicine Rationality and Experience. Good enfrenta uma série de problemas cruciais de antropolo- 
gia médica por meio de uma abordagem interpretativa que, como ele mesmo afirma: "mantém uma conversa aberta com a teoria crítica” (Good, 1994:63).

\section{As implicações da teoria interpretativa na formação e prática clínica}

Esta perspectiva nos convida a repensar não somente a prática médica mas também a formação em medicina, quase sempre ancorada a uma visão biomédica e tecnicista da doença; cada encontro com o paciente tem uma dimensão técnica mas também experiencial e ética. A hermenêutica abre novos caminhos na formação e na prática médica, modificando seus objetivos e suas finalidades, colocando a necessidade de uma nova compreensão da dimensão experiencial e do sofrimento do paciente. Este segundo caminho incorpora a dimensão subjetiva do paciente, assim como a dimensão social, elementos importantes em diferentes aspectos da prática clínica como no encontro médico-paciente (Skultans, 1998). Como apresentamos em um artigo publicado: "Gadamer conduz a reflexão sobre a humanização da medicina, em particular da relação do médico com o paciente, para o reconhecimento da necessidade de uma maior sensibilidade diante do sofrimento do paciente. Esta proposta, em relação a qual várias outras convergem, aspira pelo nascimento de uma nova imagem profissional, responsável pela efetiva promoção da saúde, ao considerar o paciente em sua integridade física, psíquica e social, e não somente de um ponto de vista biológico" (Caprara \& Franco, 1999:648).

Um primeiro aspecto se refere à ética da profissão, à atitude de respeito frente aos colegas e aos pacientes, às qualidades morais que o médico precisa ter. Segundo Dawnie et al. (2000), essas qualidades podem ser aprendidas em um processo de formação por meio de quatro formas diferentes: (1) A leitura e discussão de textos de filosofia moral; (2) A discussão de casos que introduzem na discussão aspectos éticos; (3) A utilização de um diário de campo, por parte dos médicos, para registrar casos "particulares" que enfrentam na atividade de consultório; (4) A utilização da arte, principalmente da literatura e de peças teatrais.

As obras literárias permitem abordar aspectos da vida humana, como o fato de saber lidar com as emoções, que são elementos constitutivos importantes especialmente na relação médico-paciente. Como afirma Scliar (1996), a relação médico-paciente é inevitavelmente colorida pela emoção, pela angústia muitas ve- zes. Mas o problema está ligado ao fato de que o texto médico se expressa por meio de uma linguagem simples, neutra, que pretende evitar as emoções. As frases dos artigos científicos são constituídas por sujeitos impessoais, sem incredulidade, sem dor, sem paixão. É por isso que a ansiedade médica frente ao sofrimento, à morte, à doença, procura o texto literário, a ficção, a poesia como formas de expressão. A comunicação médico-paciente pode ser aprendida como técnica, mas certamente a obra literária permite construir um contexto ético no qual a relação vai se desenvolvendo. O médico tem de entender o paciente de um ponto de vista científico, utilizando os instrumentos e os conhecimentos da literatura de maneira que ele possa tomar as melhores decisões no processo diagnóstico e terapêutico. Estas ações, tomadas valendo-se da medicina baseada nas evidências, são necessárias mas não suficientes. O médico tem de adequar sua intervenção clínica considerando o paciente enquanto sujeito, tomando em conta a experiência da doença, as percepções do paciente, adquirindo uma sensibilidade e uma capacidade de escuta que vão além da dimensão biológica. Estes aspectos podem ser desenvolvidos somente por intermédio de um processo de formação mais abrangente, que incorpore elementos das ciências humanas no desenvolvimento educacional (Simpson et al., 1991).

Nas doenças crônicas (nas quais a biomedicina oferece somente respostas parciais), a medicina clínica pode responder melhor aos pacientes, incorporando no tratamento uma análise da experiência do sujeito. Isso poderia ajudar a evitar prescrições inadequadas e excessivas de medicamentos. Uma visão restrita dos fatores causais, focalizada exclusivamente nos aspectos biológicos, é inadequada para compreender o papel dos fatores psicossociais na etiologia e como estes se integram às causas físicas.

Uma compreensão mais integrada pode produzir benefícios na parte diagnóstica também. Mesmos se estes benefícios são plausíveis, têm de ser demostrados e neste campo ainda muito tem de ser feito. As evidências também têm de ser adequadas a uma nova e mais rica concepção de que tipo de evidências são pertinentes na avaliação clínica. Trata-se de um campo que precisa de investigações e elaborações conceituais e empíricas. A medicina tem de saber lidar com personalidades, com expectativas, medos, ansiedades - além da dimensão biológica do funcionamento do corpo humano. 


\section{Agradecimentos}

Gostaria de agradecer a Naomar de Almeida Filho pela leitura atenta e crítica de uma primeira versão deste texto. Meu reconhecimento também a Ana Angela Farias pela ajuda na edição deste artigo. Enfim, aos alunos do curso de Antropologia da Saúde do Instituto de Saúde Coletiva da Universidade Federal da Bahia, do Mestrado em Saúde Comunitária da Universidade Federal do Ceará e do Mestrado em Saúde Pública da Universidade Estadual do Ceará pela discussão aberta e construtiva de diferentes idéias expressas neste texto, mesmo afirmando que as considerações aqui apresentadas são de minha exclusiva responsabilidade.

\section{Referências}

ALMEIDA FILHO, N., 2001. For a general theory of health: Preliminary epistemological and anthropological notes. Cadernos de Saúde Pública, 17: 753-799.

BARRY, C. A.; STEVENSON, F.; BRITTEN, N.; BARBER, N. \& BRADLEY, C., 2001. Giving voice to the lifeworld. More humane, more effective medical care? A qualitative study of doctor-patient communication in general practice. Social Science and Medicine, 4:487-505.

BENJAMIN, W.; HORKHEIMER, M.; ADORNO, T. \& HABERMAS, J., 1983. Textos Escolhidos. São Paulo: Editor Victor Civita.

BIBEAU, G., 1981. The semantic network in ngbandi disease nosology. Social Science and Medicine, Part B, 15:295-307.

BIBEAU, G., 1992. Entre Sens et Sens Commun. Ottawa: Société Royale du Canada.

BIBEAU, G., 1994. Hay una enfermedad en las Americas? Otro camino de la antropología médica para nuestro tiempo. In: Cultura y Salud en la Construcción de las Américas, Anales, pp. 44-70, Bogotá: Instituto Colombiano de Cultura, Universidad de los Andes.

BIRMAN, J., 1991. Interpretação e representação na saúde coletiva. Physis, 2:7-22.

BOURDIEU, P., 1980. Le Sens Pratique. Paris: Minuit.

BREIHL, J. \& GRANDA, E., 1989. Epidemiologia y contrahegemonia. Social Science and Medicine, 11: 1121-1127.

CAPRARA, A. \& FRANCO, L. S. A., 1999. A relação paciente-médico. Para uma humanização da prática médica. Cadernos de Saúde Pública, 15:647-654.

CORIN, E., 1992. Le Jeu de la Différence. Ottawa: Société Royale du Canada.

CORIN, E., 1995. The culture frame: Context and meaning in the construction of health. In: Society and Health (B. C. Amick, S. Levine, A. R. Tarlov \& D. C. Walsh, ed.), pp. 272-304, Oxford: Oxford University Press.
CORIN, E.; BIBEAU, G. \& UCHÔA, E., 1993. Eléments d'une sémiologie anthropologique des troubles psychiques chez les Bambara, Soninké et Bwa du Mali. Anthropologie et Société, 1-2:125-156.

CZERESNIA, D., 1999. The concept of health and the difference between prevention and promotion. Cadernos de Saúde Pública, 15:701-709.

DAWNIE, R. S.; MACNAUGHTON, J. \& RANDALL, F., 2000. Clinical Judegment. Evidence in Practice. Oxford: Oxford University Press.

DREYFUS, H. L., 1991. Being in the World. A Commentary on Heidegger's Being and Time. Cambridge: Mit Press.

DREYFUS, H. L. \& ZIMMERMAN, M., 1991. Applied Heidegger. Evanston: North-Western University Press.

EVANS, M. \& SWEENEY, K., 1999. Exploring the medical humanities. BMJ, 319:1216.

FOUCAULT, M., 1977. O Nascimento da Clínica. Rio de Janeiro: Forense Universitária.

FRANKENBERG, R., 1988. Gramsci, culture and medical anthropology: Kundry and parisfal? Or rat's tail to sea serpent? Medical Anthropology Quarterly, 2:324-337.

GADAMER, H.-G., 1994. Dove si Nasconde la Salute. Milano: Raffaello Cortina Editore.

GADAMER, H.-G., 1997. Verdade e Método. Petrópolis: Vozes.

GEANELLOS, R., 2000. Exploring Ricoeur's hermeneutic theory of interpretation as a method of analysing research texts. Nursing Inquiry, 7:112119.

GEERTZ, C., 1983. Local Knowledge. Further Essays in Intrepretive Anthropology. New York: Basic Books.

GOOD, B., 1994. Medicine, Rationality and Experience. An Anthropological Perspective. Lewis Henry Morgan Lectures. Cambridge: Cambridge University Press.

HABERMAS, J., 1976. Connaissance et Intérêt. Paris: Gallimard. 
HABERMAS, J., 1984. The Theory of Communicative Action. Reason and the Rationalization of Society, v.1, London: Heinemann.

HEIDEGGER, M., 1976. Essere e Tempo. Milano: Longanesi.

HEIDEGGER, M., 1977. Sein und Zeit. Tübingen: Max Niemeyer Verlag.

KIERKEGAARD, S., 1952. La Malattia Mortale. Milano: Edizioni di Comunitá.

KIERKEGAARD, S., 1991. Il Concetto di Angoscia. Firenze: Sansoni.

KLEINMAN, A., 1988. The Illness Narratives. New York: Basic Books.

LAURELL, A. C., 1989. Social Analysis of collective health in Latin America. Social Science and Medicine, 11:1183-1191.

MARTINEZ-HERNAEZ, A., 1998. L'antropologia del sintomo. Fra ermeneutica e teoria critica. $A M R i$ vista della Società Italiana di Antropologia Medica, 5-6:7-37.

McKEOWN, T., 1979. The Role of Medicine. Oxford: Basil Blackwell.

MISHLER, E. G., 1984. The Discourse of Medicine. The Dialectics of Medical Interviews. Norwood: Ablex.

MORE, A. R., 1976. Medical humanities - A new medical adventure. New England Journal of Medicine, 295:1479-1480.

NAVARRO, V., 1983. Radicalism, marxism and medicine. International Journal of Health Services, 13: 179-202.

PANDOLFI, M., 1990. Boundaries inside the body: Women's sufferings in Southern peasant Italy. Culture Medicine and Psychiatry, 2:255-273.

RICOEUR, P., 1994. Tempo e Narrativa. Campinas: Papirus.

ROBERTSON-MALT, S., 1999. Listening to them and reading me: A hermeneutic approach to understanding the experience of illness. Journal of Advanced Nursing, 2:290-297.
SCHLEIBEL, I., 2000. Gadamer Between Heidegger and Habermas. Boston: Rowman \& Littlefield Publishers.

SCLIAR, M., 1996. A Paixão Transformada. Rio de Janeiro: Companhia das Letras.

SIMPSON, M.; BUCKMAN, R.; STEWART, M.; MAGUIRE, P.; LIPKIN, M.; NOVACK, D. \& TILL, J., 1991. Doctor-patient communication: The Toronto consensus statement. BMJ, 303:1385-1387.

SKULTANS, V., 1998. Anthropology and narrative. In: Narrative Based Medicine. Dialogue and Discourse in Clinical Practice (T. Greenhalgh \& B. Hurwitz, ed.), pp. 225-233, London: BMJ Books.

SVENAEUS, F., 1999. The Hermeneutics of Medicine and the Phenomenology of Health: Steps Towards a Philosophy of Medical Practice. Linkoping: Linkoping University.

TAMBIAH, S. J., 1985. Culture, Thought and Social Action. Cambridge: Harvard University Press.

TODRES, L. \& WHEELER, S., 2001. The complementarity of phenomenology, hermeneutics and existentialism as a philosophical perspective for nursing research. International Journal of Nursing Studies, 38:1-8.

VIRCHOW, R., 1985. Report on the typhus epidemic en upper silesia, In: Collected Essays on Public Health and Epidemiology (L. J. Rather, ed.), p. 311, Canton: Science History Publications.

WIDDERSHOVEN, G., 1999. Care, cure and interpersonal understanding. Journal of Advanced Nursing, 5:1163-1169.

WIKLUND, L.; LINDHOLM, L., \& LINDSTROM, U. A., 2002. Hermeneutics and narration: A way to deal with qualitative data. Nursing Inquiry, 9:114-125.

WULFF, H. R.; PEDERSEN, S. A. \& ROSENBERG, R., 1995. Filosofia della Medicina. Milano: Raffaello Cortina Editore.

Recebido em 14 de dezembro de 2001

Versão final reapresentada em 12 de dezembro de 2002 Aprovado em 20 de dezembro de 2002 